\title{
Preface to measurement, specification and forecasting of the Solar Energetic Particle (SEP) environment and Ground Level Enhancements (GLEs)
}

\author{
Alexander Mishev ${ }^{1,2, *}$, and Piers Jiggens ${ }^{3}$ \\ ${ }^{1}$ Space Climate Research Unit, University of Oulu, Finland \\ 2 Sodankylä Geophysical Observatory, University of Oulu, Finland \\ ${ }^{3}$ European Research and Technology Centre, European Space Agency, Noordwijk, The Netherlands
}

Received 19 December 2018 / Accepted 14 January 2019

\begin{abstract}
The Sun emits energetic particles following eruptive events such as solar flares and Coronal Mass Ejections (CMEs). Solar Energetic Particles (SEPs) arrive in bursts known as Solar Particle Events (SPEs), which penetrate into the Earth's magnetosphere. SEPs with large enough energy induce a complicated atmospheric cascade, which secondary particles lead to an enhancement of count rate of ground-based detectors e.g. Neutron Monitors (NMs). This class of SEPs is therefore referred as Ground Level Enhancements (GLEs). The characterisation of the high-energy SEPs environment with corresponding space weather effects is important for space flights, aviation, and satellite industry. In this topical issue recent developments, addressing important user needs in the space radiation environment domain are published. Some articles are relevant to the specification of the SEP environment whilst others focus on space weather prediction of SEP fluxes. Catalogues based on measurement and processing of SEPs including ground-based data, and modelling of aircrew radiation exposure during major events are also presented.
\end{abstract}

Keywords: Solar Energetic Particles / GLE events / particle radiation environment / space weather

\section{Introduction}

Solar Energetic Particles (SEPs) are produced during eruptive events at the Sun such as solar flares and Coronal Mass Ejections (CMEs) (e.g. Reames, 2013; Desai \& Giacalone, 2016, and references therein). They consists of electrons, protons and heavier ions with kinetic energies ranging from tens of $\mathrm{keV}$ to about $10 \mathrm{GeV}$. Considerable enhancements of SEP flux by several orders of magnitude above the background are known as Solar Particle Events (SPEs). Those events are among the most important drivers of space weather (e.g. Lilensten \& Bornarel, 2009, and references therein). High energy SEPs may lead to a considerable harm to space missions. Protons in the 1-10 MeV/nucleon range are important drivers of solar array performance degradation whilst $>30 \mathrm{MeV} /$ nucleon protons are a key factor in electronic component degradation through ionising doses deposited in semi-conductors. Higher energy protons and heavy ions induce single event effects disrupting the performance of electronics or, in extreme cases, irreparably damaging them. SEPs also contribute to radio

\footnotetext{
*Corresponding author: alex_mi.shev@yahoo.com
}

blackouts, specifically in polar and sub-polar regions and their enhancing of the radiation environment in the vicinity of Earth and Earth's atmosphere, poses a threat to astronauts and even aircrew and passengers especially on polar flights paths (e.g. Vainio et al., 2009, and references therein).

Usually the energy of SEPs only extends to several tens of $\mathrm{MeV} /$ nucleon. However, occasionally solar protons are accelerated to few $\mathrm{GeV} /$ nucleon producing a complicated nuclearelectromagnetic-muon shower of secondary particles in the Earth's atmosphere, which reaches the ground and increase the count rates of ground based Neutron Monitors (NMs) (Hatton, 1971; Grieder, 2001). This class of SPEs is known as Ground Level Enhancements (GLEs), which occur with a rate roughly one per year (Shea \& Smart, 1990; Stoker, 1995; Klein \& Dalla, 2017). During solar maximum and declining phase of the solar cycle major SPEs and GLEs can drastically change the Earth's radiation environment leading to increased absorbed dose at space, sub-orbital and even aviation flight altitudes (e.g. Shea \& Smart, 2000).

Therefore, characterisation of the SEP environment, occurrence probability, peak fluxes and fluence with the corresponding effects is important for various industry sectors including 
space flights, satellite design and operations and civil aviation. In order to properly assess various space weather effects due to SEPs, a detailed study and modelling of their characteristics, effects and environment are necessary. In this volume several recent achievements in the field are presented, based on experimental data with the corresponding analysis and modelling.

\section{Summary of measurement, specification and forecasting of SEP environment}

In order to derive the necessary information of cosmic rays and energetic particles, specifically SEPs near Earth, it is necessary to provide a good basis for their study, usually based on statistical analysis (e.g. Kudela, 2016, and references therein). Therefore, development of new catalogues providing information for SEP events near the Earth, covering one and/or several solar cycles and wide energy range is very important.

In this volume, the paper of Paassilta et al. (2017) presents a new catalogue of SEP events, which covers solar cycle 23 and solar cycle 24, the latter from 1996 to 2016. In total 176 SEP events are presented with their onset and solar release times, the latter estimated on the basis of velocity dispersion analysis and time-shifting analysis for protons, accordingly time-shifting analysis for near-relativistic electrons. The catalogue is based on 55-80 MeV proton data derived by SOHO/ERNE, within ERNE proton and heavy ion observations and ACE/EPAM data for near-relativistic electrons, SOHO/EPHIN for relativistic electrons, SOHO/LASCO for CMEs and GOES soft X-rays data. According to the performed statistical analysis it was shown that the majority of CMEs associated with SEP events were of halo type with greater proportion during solar cycle 24. A possible explanation can be attributed to the lower total pressure in the heliosphere, which allows more CMEs to become halo events.

An important achievement based on a previously published catalogue is reported by Papaioannou et al. (2018), where the occurrence probability of SEP events in the vicinity of Earth within the peak fluxes and fluence as a function of CME parameters, namely linear speed $V$ and the Angular Width (AW) is assessed. It was shown that the occurrence probability strongly depends on the CME speed $V$ and the AW e.g. the highest association of $72.70 \%$ is obtained for full halo CMEs with $V \geq 1500 \mathrm{~km} \mathrm{~s}^{-1}$, while the lowest of $0.7 \%$ is derived for non halo CMEs with $V$ in the range $400 \mathrm{~km}-1000 \mathrm{~km} \mathrm{~s}^{-1}$. Accordingly, the SEP occurrence probabilities are different as much as 25.55 times according to the CME speed $V$, comparing fast versus slow CMEs, respectively 43.78 times using $\mathrm{AW}$, comparing halo to non-halo CMEs. Those findings are used to build a module for operational purposes FORecasting Solar Particle Events and Flares (FORSPEF, Anastasiadis et al., 2017), which performs a short term nowcasting of SEP events using near real-time information from CACTus (Bonte et al., 2011).

In the paper of Robinson et al. (2018), a new database of proton episode-integrated fluences, based on IMP8 and GOES records is presented. This record of SPEs spans from 1976 to 2016, and therefore is one of the largest continuous records of episode-integrated SEP fluence. A good agreement with the European Space Agency's solar energetic particle environment model Solar Energetic Particle Environment Modelling (SEPEM) Reference Data Set (RDS) is observed, especially at lower energies (5-50 MeV/nucleon). The differences in the higher energy are due most-likely to the processing procedure applied to GOES data.

In Jiggens et al. (2018), the Solar Accumulated and Peak Proton and Heavy Ions Radiation Environment (SAPPHIRE) model is presented. The model provides outputs for all aspects relevant to the specification of the SEP environment in the energy range from $0.1 \mathrm{MeV}$ to $1 \mathrm{GeV}$, for the derivation of effects on spacecraft. The model provides peak flux, event fluence and mission accumulated fluence for periods of solar maximum and solar minimum. SAPPHIRE is compared with various models CREME96, ESP-PSYCHIC and JPL and ACE/EPAM data. The high energy part is compared with a model based on GLE data presented in this volume by Raukunen et al. (2018).

The catalogues and fluence models of SEP events allows one the develop tools for assessment of their environment in space and/or the verify the existing models, specifically aimed to assess the radiation environment. The paper of Raukunen et al. (2018) describes two solar proton fluence models based on analysis of GLEs observed by NMs. Over the years NMs have been successfully used for estimation of spectral and angular characteristics of high energy SEPs near Earth using records from the global network (e.g. Bieber \& Evenson, 1995). The models use data retrieved over solar cycles 19-24. The first model uses GLE flunces, which have been derived by use of a four-parameter double power law function (Band function) in order to fit the particle rigidity in the range from $10 \mathrm{MeV} /$ nucleon to $1 \mathrm{GeV} /$ nucleon. The second model generates SPEs by randomly sampling the Band function parameters based on two independent and two dependent variables. The paper is an important achievement for the community, since it presents a list of spectral parameters during most of the GLE events. In addition a reasonable agreement between the presented models and JPL, ESP and SAPPHIRE models is reported.

A new probabilistic model, namely the Virtual Enhancements-Solar Proton Event Radiation (VESPER) model (Aminalragia-Giamini et al., 2018) introduces a novel paradigm for the modelling of SEP environment at $1 \mathrm{AU}$. The model is based on SEPEM RDS and produces virtual time-series of proton fluxes (Crosby et al., 2015). It differs from the existing models, which are mostly based on probabilistic descriptions of SEP characteristics e.g. peak flux and fluence. The model output allows one straightforwardly to compute SEP radiation effects in terms of time-series.

A new class of high energy SEP events was recently introduced, namely sub-GLEs (Atwell et al., 2015; Paassilta et al., 2017). According to the recently proposed definition Poluianov et al. (2017), a sub-GLE event is registered when it is observed a statistically significant increase by at least two differently located high-altitude NM stations, but without a response of near sea level NMs, while for the registration of a GLE is required a count rate increase of at least two differently located NMs and at least one located near sea level. Whilst having softer spectra than GLEs, sub-GLEs have harder spectra than 
other SPEs with detectable fluxes of particles with energies higher than $300 \mathrm{MeV} /$ nucleon (e.g. Atwell et al., 2015; Paassilta et al., 2017).

In the work of Mishev et al. (2017), several candidates for sub-GLE events are studied. On the basis of worldwide NM database records and a convenient optimization procedure, the spectral and angular characteristics of sub-GLE particles are assessed. Subsequently, using a recent model (Mishev \& Usoskin, 2015) the radiation exposure at typical commercial flight cruise altitudes of 35000 feet above sea level during these events is estimated. This further demonstrates that the global NM can be used to address important space weather issues related to alert signals and assessment of radiation environment in near Earth space and Earth's atmosphere (e.g. Mavromichalaki et al., 2011).

The second strongest GLE ever observed was the event of 20 January 2005. In the work Kalegaev et al. (2018) is derived the near-Earth radiation environment by use of observed solar wind and interplanetary magnetic field parameters in computations with the A2000 magnetospheric model. It was shown that during the early, very anisotropic phase of the event, particle penetration into the magnetosphere took place in the regions where the magnetospheric and interplanetary magnetic field vectors are parallel. It was demonstrated that two regions on the magnetopause are responsible for particle access to the magnetosphere: The near equatorial day-side region and open field lines window at the high-latitude magnetospheric boundary.

In a review by Miroshnichenko (2018) are considered both modern state problems in the field of GLE physics and applications, and some historical achievements with the corresponding critical analysis. In the paper are reviewed GLE properties as total statistics, occurrence rate, longitude distribution, ranking and specific GLEs are discussed in details. An important space weather issue related to the radiation hazard in space and in the Earth's atmosphere is discussed, where both early and recent models are presented. The problem with extreme SPEs is briefly mentioned. Here we would like the stress that the inclusion of Nitrates as a proxy for past extreme SPEs is included only for illustration and historical reasons, represents author's personal point of view, because this method is not suitable for scientific analysis according to recent study by Mekhaldi et al. (2017).

\section{Conclusions}

This volume is comprised of a set of articles with recent findings in the field of space weather and space physics, related to the measurements and specification of SEP environment. The presented studies facilitate the characterisation of the highenergy solar particles environment enabling estimation of the related space weather effects. The models and catalogues presented herein are relevant to the specification of the SEP environment including aircrew exposure to radiation, as well as the specification and prediction of SEP fluxes based on both space-borne and ground based measurement, encompassing most of the present issues related to the dynamic energetic particle environment in the vicinity of Earth and within its atmosphere. These studies give a good basis for further development of tools for mitigation of the radiation hazards in space due to SEPs.
Several studies presented here used records from the global NM network. Besides their use in alerts and forecast of SEP events, the analysis of these data can be used to estimate the spectra of GLEs for specification and near-real-time reconstruction of the radiation environment in space and at aircraft altitudes. This capability is very challenging using only spacebased measurements. Nowadays, the existence and continuous functioning of several NM stations is questionable, therefore the support to the global NM network from the corresponding scientific funds, governments and non the least the space flight operators is crucial.

Acknowledgements. The guest Editors in chief of this volume A. Mishev and P. Jiggens, warmly acknowledge the guest editors: M. Dierckxsens, M. Laurenza, A. Papaioannou and R. Vainio for their tremendous work as well as all the reviewers.

\section{References}

Aminalragia-Giamini S, Sandberg I, Papadimitriou C, Daglis I, Jiggens P. 2018. The virtual enhancements-solar proton event radiation (VESPER) model. J Space Weather Space Clim 8, A06. DOI: $10.1051 / \mathrm{swsc} / 2017040$.

Anastasiadis A, Papaioannou A, Sandberg I, Georgoulis M, Tziotziou K, Kouloumvakos A, Jiggens P. 2017. Predicting flares and solar energetic particle events: The FORSPEF tool. Solar Phys 292(9), 134. DOI: 10.1007/s11207-017-1163-7.

Atwell W, Tylka A, Dietrich W, Rojdev K, Matzkind C. 2015. SubGLE solar particle events and the implications for lightly-shielded systems flown during an era of low solar activity. In: 45th International Conference on Environmental Systems, Bellevue, WA, 12-16 July 2015, 1-12. ICES-2015-340.

Bieber J, Evenson P. 1995. Spaceship earth - An optimized network of neutron monitors. In: Proc. of 24th ICRC, vol. 4, 28 August-8 September 1995, Rome, Italy, 1316-1319

Bonte K, Jacobs C, Robbrecht E, de Groof A, Berghmans D, Poedts S. 2011. Validation of CME detection software (CACTus) by means of simulated data, and analysis of projection effects on CME velocity measurements. Solar Phys 270, 253-272. DOI: 10.1007/s11207-011-9740-7.

Crosby N, Heynderickx D, Jiggens P, Aran A, Sanahuja B, et al. 2015. SEPEM: A tool for statistical modeling the solar energetic particle environment. Space Weather 13(7), 406-426. DOI: 10.1002/2013SW001008.

Desai M, Giacalone J. 2016. Large gradual solar energetic particle events. Living Rev Solar Phys 13(1), 3. DOI: 10.1007/s41116-0160002-5

Grieder P. 2001. Cosmic rays at Earth researcher's reference manual and data book, Elsevier Science, Amsterdam, The Netherlands. ISBN 978-0-444-50710-5.

Hatton C. 1971. The neutron monitor. In: The neutron monitor progress in elementary particle and cosmic-ray physics, vol. X, North Holland Publishing Co., Amsterdam, The Netherlands. (Chap. 1).

Jiggens P, Heynderickx D, Sandberg I, Truscott P, Raukunen O, Vainio R. 2018. Updated model of the solar energetic proton environment in space. J Space Weather Space Clim 8, A31. DOI: $10.1051 / \mathrm{swsc} / 2018010$

Kalegaev V, Vlasova N, Nazarkov I, Melkova S. 2018. Magnetospheric access for solar protons during the January 2005 SEP event. J Space Weather Space Clim 8, A55. DOI: 10.1051/swsc/ 2018040 
Klein K-L, Dalla S. 2017. Acceleration and propagation of solar energetic particles. Space Sci Rev 212(3-4), 1107-1136. DOI: 10.1007/s11214-017-0382-4.

Kudela K. 2016. On low energy cosmic rays and energetic particles near Earth. Contrib Astron Obs Skaln Pleso 46(1), 15-70.

Lilensten J, Bornarel J. 2009. Space weather, environment and societies, Springer, Dordrecht, The Netherlands. ISBN 978-1-40204332-1.

Mavromichalaki H, Papaioannou A, Plainaki C, Sarlanis C, Souvatzoglou G, et al. 2011. Applications and usage of the real-time Neutron Monitor Database. Adv Space Res 47, 2210-2222.

Mekhaldi F, McConnell J, Adolphi F, Arienzo M, Chellman N, Maselli O, Moy A, Plummer C, Sigl M, Muscheler R. 2017. No coincident nitrate enhancement events in polar ice cores following the largest known solar storms. J Geophys Res: Atmos 122(21), 11.900-11.913. DOI: 10.1002/2017JD027325.

Miroshnichenko L. 2018. Retrospective analysis of GLEs and estimates of radiation risks. J Space Weather Space Clim 8, A52. DOI: $10.1051 / \mathrm{swsc} / 2018042$.

Mishev A, Poluianov S, Usoskin I. 2017. Assessment of spectral and angular characteristics of sub-GLE events using the global neutron monitor network. J Space Weather Space Clim 7, A28. DOI: $10.1051 /$ swsc/2017026.

Mishev A, Usoskin I. 2015. Numerical model for computation of effective and ambient dose equivalent at flight altitudes: Application for dose assessment during GLEs. J Space Weather Space Clim 5(3), A10. DOI: 10.1051/swsc/2015011.

Paassilta M, Raukunen O, Vainio R, Valtonen E, Papaioannou A, et al. 2017. Catalogue of $55-80 \mathrm{MeV}$ solar proton events extending through solar cycles 23 and 24. J Space Weather Space Clim 7, A14. DOI: 10.1051/swsc/2017013.

Papaioannou A, Anastasiadis A, Sandberg I, Jiggens P. 2018. Nowcasting of solar energetic particle events using near real-time coronal mass ejection characteristics in the framework of the FORSPEF tool. J Space Weather Space Clim 8, A37. DOI: $10.1051 / \mathrm{swsc} / 2018024$

Poluianov S, Usoskin I, Mishev A, Shea M, Smart D. 2017. GLE and Sub-GLE redefinition in the light of high-altitude polar neutron monitors. Solar Phys 292(11), 176. DOI: 10.1007/s11207-0171202-4.

Raukunen O, Vainio R, Tylka A, Dietrich W, Jiggens P, Heynderickx D, Dierckxsens M, Crosby N, Ganse U, Siipola R. 2018. Two solar proton fluence models based on ground level enhancement observations. J Space Weather Space Clim 8, A04. DOI: $10.1051 / \mathrm{swsc} / 2017031$.

Reames D. 2013. The two sources of solar energetic particles. Space Sci Rev 175(1-4), 53-92. DOI: 10.1007/s11214-013-9958-9.

Robinson Z, Adams J, Xapsos M, Stauffer C. 2018. Database of episode-integrated solar energetic proton fluences. $J$ Space Weather Space Clim 8, A24. DOI: 10.1051/swsc/2018013.

Shea M, Smart D. 1990. A summary of major solar proton events. Solar Phys 127, 297-320.

Shea M, Smart D. 2000. Cosmic ray implications for human health. Space Sci Rev 93(1-2), 187-205.

Stoker P. 1995. Relativistic solar proton events. Space Sci Rev 73(3-4), 327-385. DOI: 10.1007/BF00751240.

Vainio R, Desorgher L, Heynderickx D, Storini M, Flückiger E, et al. 2009. Dynamics of the Earth's particle radiation environment. Space Sci Rev 147(3-4), 187-231.

Cite this article as: Mishev A \& Jiggens P 2019. Preface to measurement, specification and forecasting of the Solar Energetic Particle (SEP) environment and Ground Level Enhancements (GLEs). J. Space Weather Space Clim. 9, E1. 